\title{
Mitteilungen aus der Bundeskonferenz Deutscher Kliniken für Psychiatrie und Psychotherapie (BDK)
}

Verantwortlich für diese Rubrik: Gerhard Längle, Tübingen/Bad Schussenried; Thomas Pollmächer, Ingolstadt

\author{
Versorgung von Menschen mit \\ geistiger Behinderung und \\ psychischen Störungen \\ $\nabla$
}

Bericht des AK Geistige Behinderung der BDK

Der Arbeitskreis (AK) hat sich zur Aufgabe gemacht, die medizinisch-psychiatrische Versorgung von Menschen mit geistiger Behinderung in Deutschland zu verbessern. Er vernetzt eine Spannbreite von Versorgungsangeboten, darunter 33 Spezialbereiche in Psychiatrischen Kliniken und 3 Spezialkliniken. Mit dem „Wandern“ der Tagungen, zu denen jedes Mal von einem anderen Mitglied des Arbeitskreises eingeladen wird, ist ein Kennenlernen der Diversität unterschiedlichster Anpassungsstrategien an regionale Bedingungen möglich. Außerdem erwirbt der AK in seiner multiprofessionellen Zusammensetzung und interdisziplinären Ausrichtung kontinuierlich einen Zuwachs an Expertise in spezifischen Fragen der Diagnostik und Behandlung, aber auch in Fragen der Ökonomie und Qualitätssicherung sowie Organisationsentwicklung. Dies konnte in der Vergangenheit für zahlreiche Projekte vor Ort nutzbar gemacht werden. Den einladenden Kliniken gebührt ein besonderer Dank für ihre Offenheit und Bereitschaft, die Erfahrung im eigenen Haus zu teilen. Die besuchten Bereiche/Kliniken profitieren meist von einem hohen persönlichen Einsatz der Mitarbeiter und der Sensibilität für die Belange dieser Zielgruppe im oberen Management. Nichtsdestotrotz bedarf es der Veränderung ungünstiger Rahmenbedingungen, die heute häufig noch Professionalität und Qualität beeinträchtigen, um die Versorgung von Menschen mit geistiger Behinderung und psychischen Störungen erhalten zu können und irgendwann eine Umsetzung der UN-Behindertenrechtskonvention für die Beteiligten spürbar werden zu lassen.

Folgende Themen befinden sich aktuell in Bearbeitung:

\section{Prävention von Gewaltaufkommen und dem Einsatz von Zwangsmitteln im stationär-psychiatrischen Kontext}

Ausgehend von dem Umstand, dass viele, vermutlich die meisten stationär-psychiatrischen Behandlungen, von Menschen mit geistiger Behinderung durch fremdund selbstverletzendes Verhalten veranlasst sind, erstaunt es zunächst nicht, wenn auch das Gewaltaufkommen auf Station zwischen den Patienten gegen Personal und Gegenstände erhöht ist und konsekutiv - im Sinne einer Gewaltspirale - der Einsatz von Zwangsmitteln steigt. Die Erfahrungen der Spezialbereiche deuten allerdings auf erhebliche Unterschiede von Einrichtung zu Einrichtung. Dies könnte mit Faktoren, wie räumlicher und personeller Ausstattung, dem Therapieangebot, aber auch mit der Definition und dem Verständnis von Begrifflichkeiten, wie "weiches Zimmer", „time out" und einem prästationär wirksamen, ambulanten Therapieangebot zusammen hängen. Eine systematische Erhebung der Rahmenbedingungen in Deutschland dazu steht aus. Der AK möchte seine Ressourcen nutzen, um eine vergleichende Erhebung durchzuführen. Ein entsprechender Fragebogen befindet sich in Vorbereitung.

Last but not least ist die psychiatrische Regelversorgung einer der letzten präventiven Interventionsmöglichkeiten in der Versorgungskette der Behandlung schwerer aggressiver Verhaltensstörungen, bevor es zu einer Anzeige mit Folge der Unterbringung im Maßregelvollzug kommt, aus dem heraus Menschen mit geistiger Behinderung schwerer, wenn überhaupt, resozialisiert werden können.

Fragen, die den AK beschäftigen, sind: Wie ist von der Psychiatrie die Brücke zu einem präventiven Ansatz einzelfallbezogen in den Einrichtungen der Behindertenhilfe, SGBXII, zu schlagen? Welcher Ergänzungen bedarf es in den Curricula der einschlägigen (heil-)pädagogischen Ausbildungsgänge, um sie auf die psychiatrischen Aspekte ausreichend vorzubereiten?
Sicher ist schon heute, dass Prävention einer Gemeinschaftsleistung vieler Parteien bedarf, wie der ambulant-psychiatrischen Versorgung, der Einrichtungen der Behindertenhilfe bis hin zur Fachaufsicht/ Heimaufsicht als Kontrollbehörde, um den Folgen, wie Gewaltaufkommen auf Station und dem Verlust der Teilhabe, entgegenzuwirken.

\section{Zur Situation von intelligenzgemin- derten Menschen im Maßregelvollzug Sensibilisierung der Träger der Behinder- tenhilfe}

In allen Maßregelvollzugseinrichtungen Deutschlands finden sich nicht unerhebliche Anteile von intelligenzgeminderten Menschen. Die Angaben schwanken zwischen ca. $6 \%$ und $25 \%$ der untergebrachten Personen (Übersicht bei Steinböck 2001). In einer Promotion versuchte Kestel (2010) zu erheben, inwieweit spezialisierte Angebote für diese Personengruppe in den Maßregelvollzugskliniken vorgehalten werden. Letztendlich fanden sich nur in 10 der 71 angeschriebenen Kliniken entsprechende spezialisierte Behandlungsbereiche. Da auch im Deliktspektrum häufig Anlassstraftaten von erheblicher Bedeutung (Sexualdelikte, Körperverletzungen, Brandstiftungen) zu finden sind und auch nicht selten eine Komorbidität, z. B. mit Alkoholmissbrauch vorliegt, handelt es sich bei dieser Personengruppe um eine sowohl therapeutisch als auch prognostisch schwierige Population. Der AK Geistige Behinderung der Bundesdirektorenkonferenz arbeitet bezüglich dieses Themas in unregelmäßigen Abständen mit dem AK Forensik zusammen; schon häufiger wurden entsprechende Spezialbereiche in Maßregelvollzugskliniken besucht und mit dem dort tätigen Personal über die spezifischen Herausforderungen diskutiert. Hierbei stellten sich, neben den üblichen Schwierigkeiten einer ausreichenden personellen Versorgung auch mit auf dem Gebiet der Arbeit mit intelligenzgeminderten Patienten vertrauten Therapeuten sowie spezialisierten Berufsgruppen, wie z.B. Heilpädagogen, auch oft 
die räumliche Ausstattung als problematisch dar. Oft fehlt es - bei hoher Fachlichkeit und persönlichem Engagement - an den Behinderungen genügend räumlicher und struktureller Erfordernisse.

Ein weiteres Thema sind mögliche präventive Ansätze, die vor allem im Bereich der Behindertenhilfe, dem Lebensumfeld, wirksam werden sollten; diese sind auch in der Nachsorge von erheblicher Bedeutung.

Im regelmäßigen Dialog der beiden AKs wird nun schon seit vielen Jahren versucht, die Situation dieser besonderen Personengruppe im Maßregelvollzug zu evaluieren und zu verbessern, stets auch im möglichst engen Kontakt zu nachsorgenden Einrichtungen der Behindertenhilfe. So ist das Frühjahrstreffen 2014 der Arbeitsgruppe erneut in Zusammenarbeit mit der Arbeitsgruppe Forensik geplant und zwar in der Christopherus Fachklinik für Forensische Psychiatrie der Alexianer in Münster am 27./28. März 2014.

\section{Abbildung des behinderungsbedingten Mehraufwands im stationären Entgelt- system}

Für die stationär-psychiatrische Behandlung gibt es einen behinderungsbedingten Mehraufwand, der sich am ehesten durch die Entwicklungsverzögerung beschreiben lässt. Er macht z. B. die Vorhaltung zusätzlicher pädagogischer bzw. heilpädagogischer Fachkräfte im multiprofessionellen Team erforderlich. Der Mehraufwand lässt sich in der derzeitigen OPSSystematik nicht abbilden. Die im aktuellen PEPP-Katalog zur Verfügung stehenden 2 Ziffern, PA01A, kalkuliert auf nur $\mathrm{n}=333$ Fälle mit eine MVD von 19,95 Tagen (1. Degressionstufe 1-8. Tag) und PA01B, kalkuliert auf $n=391$ Fällen mit einer MVD von 17,16 Tagen (1. Degressionsstufe $1-7$. Tag), bilden nicht die Versorgungsrealität ab.

Die Spezialbereiche müssen oft quer finanziert werden.

Die Kalkulation entspricht vielleicht der Realität von Patienten, die in einer Krise in die Allgemeinpsychiatrie aufgenommen und nach einer Woche entlassen werden. Die Kalkulation entspricht nicht dem Fall eines von ca. 6000 Patienten/ Jahr, der z.B. dann, ein paar Tage später, wieder in einem Spezialbereich aufgenommen wird, in der 1 . Woche erschöpft ist und sich erholt und dann zu alten Kräften und Verhaltensmustern zurückkehrt, z. B. in der 2. oder 3. Woche eine C-MRTDiagnostik in Narkose bekommt, eine apparative internistische Diagnostik in Narkose, 1:1-Betreuung und Einzeltherapie, die das Behandlungsziel nach 4-6 Wochen erreichen. Diese Kosten sind über die 5-6 Kalkulationshäuser mit Spezialbereichen zu ermitteln!

Vorschläge aus dem Arbeitskreis unterstützen eine Lösung innerhalb der OPSSystematik. Alle zuständigen Entscheidungsträger in Politik und den Verbänden, aber auch das InEK sind gefordert, eine tragbare Basis für die Realisierung eines menschenwürdigen und therapeutischen Milieus auf den Spezialstationen zu schaffen! Dies bedarf einer erhöhten Personaldichte, angemessener räumlicher Ausstattung und der Vorhaltung pädagogischer Kompetenz.

Die kommende Tagung findet am 19./20. 9.2013 auf Einladung von Herrn Dr. HohlRadke im Asklepios-Fachklinikum Brandenburg statt.

Für Fragen und Anregungen wenden Sie sich bitte an franziska.gaese@kbo.de.

Dr. Franziska Gaese

Dr. Knut Hoffmann 